\title{
Characterization of Argon-Helium Mixed Gas Inductively Coupled Plasma Based on Spectroscopic Analyses of the Argon and Zinc Emission Lines
}

\author{
Kazuaki Wagatsuma and Kichinosuke Hirokawa \\ Institute for Materials Research, Tohoku University, Katahira, Sendai 980, Japan
}

\begin{abstract}
In an Ar-He mixed gas inductively coupled plasma (ICP) the role of helium in the excitation of the zinc species was investigated through spectroscopic analyses on both the argon and zinc emission lines. Whereas the spectral widths of the argon lines were only slightly affected by helium addition, the emission intensities of the zinc lines apparently increased. Helium contributes to the increase in zinc excitation, though it causes a slight alternation of the electron density of the plasma. This results from an increment in the plasma temperatures in only the central channel where the zinc species is dominantly excited. These observations could suggest that helium acts as an energy carrier from the induction zone to the central channel of the ICP, due to the fairly high heat conductivity.
\end{abstract}

Keywords Inductively coupled plasma, argon-helium mixed gas, argon line width, zinc line intensity

Atomic emission spectrometry using inductively coupled plasma (ICP) has become widespread as a powerful analytical technique for routine works. It is well known that various parameters influence the performance of ICP spectrometry. The kind of support gas used is the most critical factor in determining the fundamental characteristics of ICP. Under ordinary operations, pure argon is usually employed in all channels of the gas flow carried to the plasma, although nitrogen ICPs are put to practical use particularly under high r.f. power conditions. ${ }^{1,2}$

Several studies have been reported concerning attempts to replace either part or all of the argon gas with

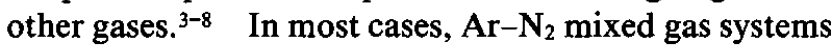
have become an object of research, since the running cost to operate the ICP could be reduced by using nitrogen as a cheaper gas. In addition, spectroscopic analyses have revealed the properties of $\mathrm{Ar}-\mathrm{N}_{2} \mathrm{ICP}$, itself, compared to those with pure argon gas, indicating that an appropriate selection of the experimental parameters can lead to the utilization of a mixed gas plasma as an analytical light source..$^{5-8}$ Helium gas has also been used as a component of mixed gas ICPs. ${ }^{7-9}$ The spectral features of the Ar-He ICP were investigated by Choot $e t$ al., who have shown that a unique advantage of this type plasma is a lower background continuum, compared to that of pure Ar ICP. ${ }^{8}$ Further, their observation of cadmium emission lines indicated that the spectral intensities in the Ar-He ICP were only slightly different from those of a pure Ar plasma. Sheppard et al. reported the use of $\mathrm{Ar}-\mathrm{He}$ mixture gas for the determination of iodine as well as the optimum conditions of the plasma param- eters. ${ }^{9}$ In their study the addition of helium was carried out in order to improve the detection limits of the less sensitive elements in pure Ar ICPs.

A line broadening due to a strong Stark effect was observed in some argon atomic lines between 500 and $600 \mathrm{~nm}^{.10}$ One can estimate the variations in the electron density in the plasma from the spectral broadening of these emission lines. ${ }^{11}$ Further, the beta line of the hydrogen atom (H I $486.1 \mathrm{~nm}$ ) is very often employed for electron density measurements, because the contribution of the Stark effect yields most parts of the entire profile of the line.

In this paper, we investigated the characteristics of the $\mathrm{Ar}-\mathrm{He}$ mixed gas ICP primarily based on an analysis of the line widths of argon emission lines. Measurements of the line widths provide knowledge regarding the plasma regions from which the argon emission lines are radiated. The induction zone of $\mathrm{ICP}^{12}$, which surrounds the central channel of the plasma, is a dominant source of the argon lines. It can therefore be expected that the spectral widths of the argon emission lines are sensitive to the state variation of the induction zone where electric energies of the r.f. power are directly transmitted. On the other hand, changes in the emission intensities of the analyte species (zinc) are caused by helium addition at the region of the central channel where sample solutions are injected. Their variations were observed under various operating conditions as a function of the helium content in the plasma gas. Our interest concerns in the role of helium in the excitation of the analytes when relatively small amounts of helium gas are mixed in pure argon plasmas. 
Table 1 Instrumentation and experimental conditions

\begin{tabular}{|c|c|}
\hline $\begin{array}{l}\text { Plasma source unit } \\
\text { R.f. generator }\end{array}$ & $\begin{array}{l}\text { ICPS-2H model (Shimadzu Corp., Japan) } \\
\text { frequency: } 27.12 \mathrm{MHz} \\
\text { nominal output power range: } 0-2.7 \mathrm{~kW} \text { (continuous rating) }\end{array}$ \\
\hline Induction coil & two turns, $30 \mathrm{~mm}$ i.d. water cooled \\
\hline Torch & Fassel-type, outer gas tube of $18 \mathrm{~mm}$ i.d. \\
\hline Nebulizer & pneumatic \\
\hline Spray chamber & Scott-type double pass \\
\hline Spectrometer & $\begin{array}{l}\text { GE- } 340 \text { model (Shimadzu Corp., Japan) } \\
\text { focal length: } 3.4 \mathrm{~m} \\
\text { grating: } 1200 \text { grooves } / \mathrm{mm} \\
\text { blaze wavelength: } 300 \mathrm{~nm} \\
\text { slit width: } 30 \mu \mathrm{m} \\
\text { slit height: } 3 \mathrm{~mm}\end{array}$ \\
\hline Photomultiplier & R-955 (Hamamatsu Photonics Corp., Japan) \\
\hline Optics & $\begin{array}{l}\text { fused-silica lens, focusing a } 1: 1 \text { image of the plasma } \\
\text { source on the entrance slit }\end{array}$ \\
\hline Gas mixer & $\begin{array}{l}\text { MX-3S model (Crown Corp., Japan) } \\
\text { Ar: } 0-251 / \mathrm{min} \text {; He: } 0-251 / \mathrm{min}\end{array}$ \\
\hline Incident r.f. power & $1.1-2.2 \mathrm{~kW}$ (as parameter) \\
\hline Reflected power & less than $40 \mathrm{~W}$, see text \\
\hline Outer gas flow rate & $16.01 / \mathrm{min}$ (total flow rate) containing $0-30 \% \mathrm{He}$ \\
\hline Intermediate gas flow rate & $1.451 / \min ($ fixed) \\
\hline Carrier gas flow rate & $1.001 / \min ($ fixed) \\
\hline Observation height & $14 \mathrm{~mm}$ ALC (fixed) \\
\hline Sample uptake rate & $c a .1 .6 \mathrm{ml} / \mathrm{min}$ when distilled water is injected \\
\hline
\end{tabular}

The effects of helium gas are thus correlated and discussed concerning the different zones of the ICP.

\section{Experimental}

\section{Apparatus}

The plasma source unit employed comprised a plasma torch, a matching box, and a radio-frequency generator. No modification of the unit was carried out. A Fastie-Ebert mounting spectrograph equipped with a photomultiplier tube was employed. Table 1 describes in detail all of the other instrumentation as well as the operating conditions.

\section{Reagents}

Zinc was selected as an analyte element. The stock solutions were made by dissolving $2.5 \mathrm{~g}$ of high purity zinc metal (more than $99.999 \%$ ) in small amounts of heated hydrochloric acid $(6 \mathrm{M})$ and by subsequent dilution to a concentration of $10 \mathrm{mg} / \mathrm{ml}$ with distilled water. Sample solutions with a zinc concentration of $0.025 \mathrm{mg} / \mathrm{ml}$ were prepared by diluting with the appropriate amounts of dilute hydrochloric acid. All of the solutions contained ca. $0.02 \mathrm{M}$ hydrochloric acid.

\section{Analytical lines}

The Zn I $213.86 \mathrm{~nm}$, the Zn II $202.55 \mathrm{~nm}$, and the Zn II $206.36 \mathrm{~nm}$ lines were selected as analytical lines for the zinc atom and ion, respectively. The literature was
Table 2 Assignment of emission lines employed

\begin{tabular}{lll}
\hline \multirow{2}{*}{ Wavelength/nm } & \multicolumn{2}{c}{ Assignment } \\
\cline { 2 - 3 } & \multicolumn{1}{c}{ Upper $/ \mathrm{eV}$} & Lower $/ \mathrm{eV}$ \\
\hline Zn I 213.86 & $5.796,4 \mathrm{~s} 4 \mathrm{p}^{1} \mathrm{P}_{1}-0.000,(4 \mathrm{~s})^{2}{ }^{1} \mathrm{~S}_{0}$ \\
Zn II 202.55 & $6.119,4 \mathrm{p}^{2} \mathrm{P}_{3 / 2}-0.000,4 \mathrm{~s}^{2} \mathrm{~S}_{1 / 2}$ \\
Zn II 206.19 & $6.011,4 \mathrm{p}^{2} \mathrm{P}_{1 / 2}-0.000,4 \mathrm{~s}^{2} \mathrm{~S}_{1 / 2}$ \\
Ar I 549.53 & $15.35,6 \mathrm{~d}[3 / 2]_{2}-13.09,4 \mathrm{p}[5 / 2]_{2}$ \\
\hline
\end{tabular}

consulted concerning any argon emission lines which exhibit relatively strong Stark broadening. ${ }^{11}$ The full widths at half maximum (FWHM) ${ }^{10}$ of the Ar I $549.53 \mathrm{~nm}$ line were observed in order to estimate the electron density in the plasma. The assignment for each line is summarized in Table 2 .

\section{Procedure}

Argon-helium mixed gases were introduced only as the outer gas flow of the ICP. For the intermediate and carrier gas flow, pure argon was employed at fixed flow rates. The total flow rate of the outer gas was kept at $161 / \mathrm{min}$ and the composition was varied from 0 to ca. $30 \% \mathrm{He}$. Because the matching networks in our instrumentation were designed for pure argon gas, it was difficult to control the reflected power appropriately at higher helium flow rates. The discharges became unstable and extinguished when the reflected power 
exceeded about $40 \mathrm{~W}$. The instrumental ability to adjust the reflected powers therefore restricted the upper limit of the He content. The allowed contents of helium were lowered along with a decrease in the r.f. incident power.

The plasma was initiated with pure argon gas. Once stable discharges were obtained, appropriate amounts of argon were gradually replaced by helium gas while any variation in the reflected power was checked.

The FWHM of the argon line was determined from duplicate individual measurements. Further, the emission intensities of the zinc lines were estimated from the average peak heights for four or five replicates. The results exhibited good precision and the relative standard deviations were within a few percent.

\section{Results and Discussion}

\section{FWHMs versus the incident radio frequency power}

In addition to the Stark effect, various line broadening effects are reflected in the line width of the argon line, since Stark broadening is not sufficiently large to overcome these contributions. ${ }^{11}$ Although it is necessary to correct for other broadening effects when the electron density is computed from the FWHMs, it is considered that the relative variations in the electron density of the plasma can be derived primarily from the uncorrected spectral widths.

It is obvious that the electron density as well as the plasma temperature increase with the supplied r.f. power. One can thus expect that the r.f. power is a significant parameter in determining the line widths. As shown in Fig. 1, we investigated the dependence of the FWHM values of the Ar I $549.5 \mathrm{~nm}$ line on the incident r.f. power.
The FWHMs increased by a factor of $c a .8$ when the power was varied from 0.8 to $2.5 \mathrm{~kW}$. A theoretical calculation by Griem ${ }^{11}$ predicts that the electron densities are of the order of $10^{15} \mathrm{~cm}^{-3}$ when the FWHM values given in Fig. 1 are used as the parameter. Such densities are not inconsistent with the results reported in a previous work $^{13}$, implying that the resultant FWHMs are governed mainly by the electron density of the plasma. Therefore, the line widths of the argon line can be employed as an indicator representing the state of the ICP.

\section{FWHMs in Ar-He mixed gas plasmas}

Figure 2 indicates the relation between the FWHM value of the Ar I $549.5 \mathrm{~nm}$ line and the helium contents in the outer gas for several incident r.f. powers. It is found that the FWHMs gradually decrease with an increase in the amount of helium when the r.f. power is less than $1.6 \mathrm{~kW}$. At higher r.f. powers, the FWHMs have a maximum point upon increasing the helium content. However, it should be noted that these changes are very slight under all of the power conditions examined. For example, the variation was within $5 \%$ of the mean FWHM value when the ICP was loaded at $2.2 \mathrm{~kW}$. Accordingly, we can conclude that the addition of helium has only little influence on the electron densities when the mixed ratio is varied up to $30 \%$. These results also suggest that in an Ar-He mixed gas ICP relatively small amounts of helium cause only a slight alternation of the plasma.

Because the induction zone of the ICP is a major source of the argon emission lines, the measurements described above may represent that helium addition scarcely affects the characteristics of the plasma core.

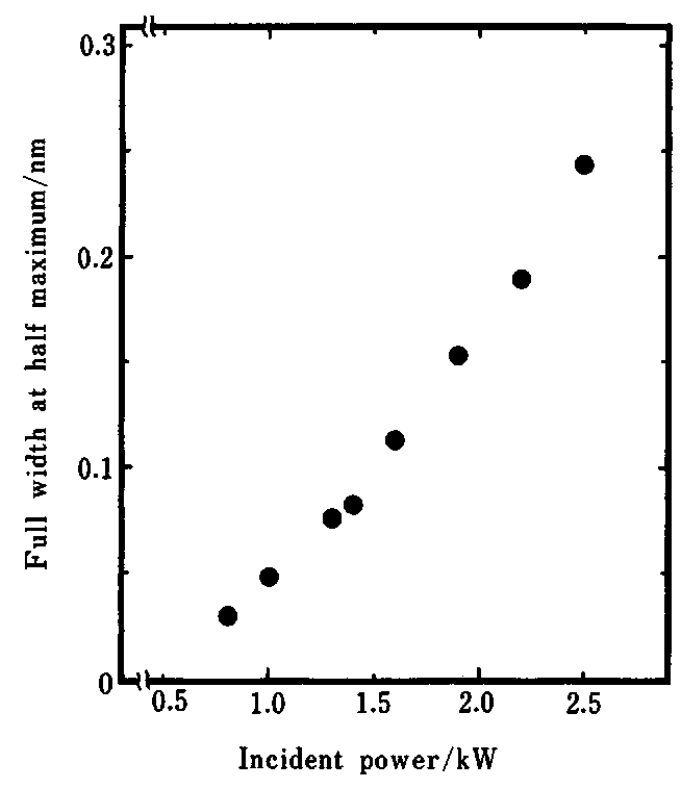

Fig. 1 Dependence of the FWHMs of the Ar I $549.5 \mathrm{~nm}$ line on the incident r.f. power.

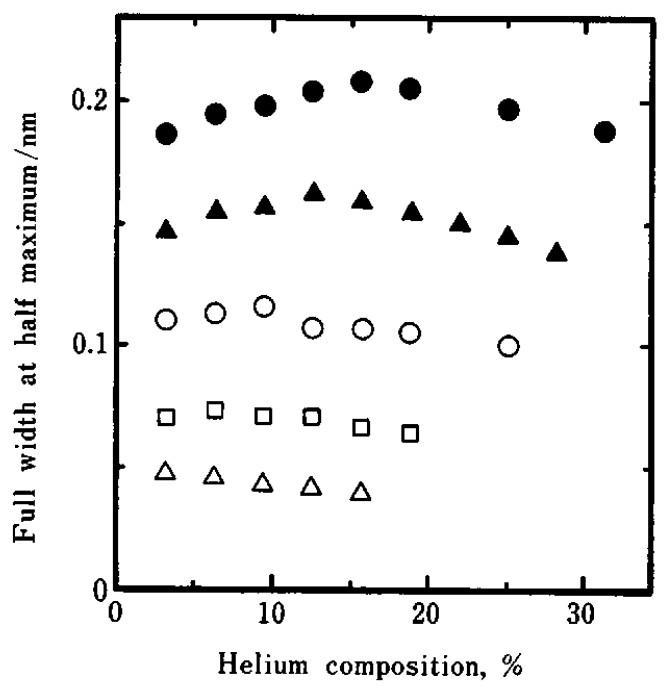

Fig. 2 Plots of the FWHMs of the Ar I $549.5 \mathrm{~nm}$ line against the helium contents in the outer gas flow for the r.f. power: $2.2 \mathrm{~kW}(\Theta), 1.9 \mathrm{~kW}(\Delta), 1.6 \mathrm{~kW}(0), 1.3 \mathrm{~kW}(\square)$ and $1.1 \mathrm{~kW}$ $(\triangle)$. 
Zinc emission intensity in Ar-He mixed gas plasmas

Figure 3(a) shows the variations in the emission intensities of the zinc emission lines as a function of the He composition in the outer gas flow. Furthermore, the intensity ratios between these lines are plotted in Fig. 3(b). The intensities increase upon increasing the helium content and then obtain a steady state. It was found that, in the case of the ionic lines, the emission intensity is doubled when the helium content is raised up to $20 \%$. The intensity variation of the ionic lines is greater compared to that of the atomic line, which results in an increase in the intensity ratio of the ionic line to the

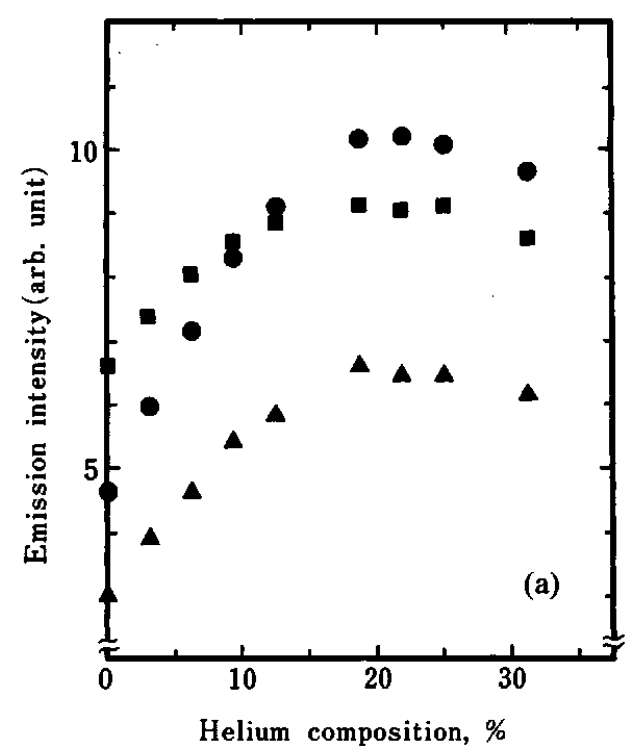

Fig. 3(a) Variation in the emission intensities of $\mathrm{Zn} \mathrm{I}$ $213.9 \mathrm{~nm}(\square), \mathrm{Zn}$ II $202.6 \mathrm{~nm}(\bullet)$, and $\mathrm{Zn}$ II $206.2 \mathrm{~nm}(\Delta)$ as a function of the helium composition in the r.f. power of $1.9 \mathrm{~kW}$.

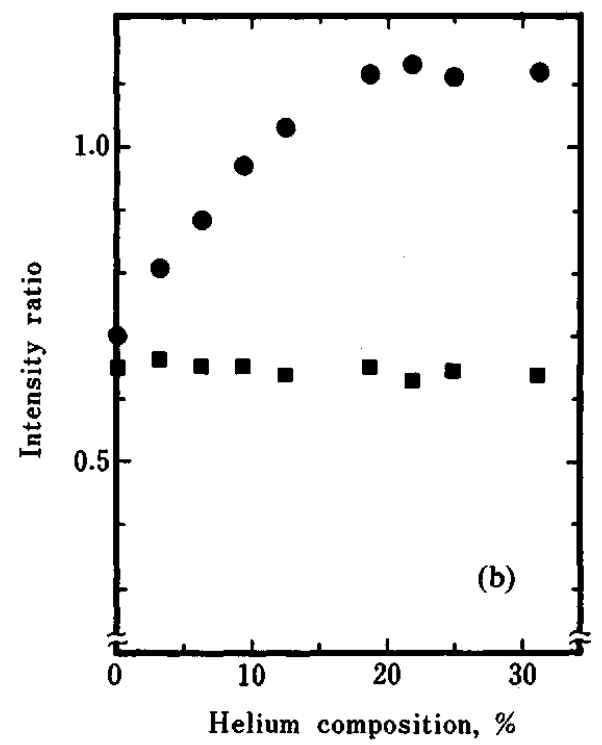

Fig. 3(b) Variation in the intensity ratios: Zn II $202.6 / \mathrm{Zn}$ I 213.9 (๑) and Zn II 206.2/Zn II 202.6 (ם). atomic line, as illustrated in Fig. 3(b). This figure also indicates that the intensity ratio between the ionic lines, whose excitation energies are almost the same, hardly changes with respect to the He mixture.

An improvement in the excitation efficiency for the zinc atomic and ionic species occurs when helium gas is added to the Ar ICP. One should notice that the variation in the ionic-to-atomic line intensity ratio is due to more active ionization in the plasma as the He content becomes higher. It can be suggested from these observations that the addition of helium gas contributes to an increment in the plasma temperature at the region of the plasma where the zinc species is excited and ionized.

Figure 4 shows the relation between the ionic-toatomic ratios of the zinc lines as well as the $\mathrm{He}$ compositions in the ICP for different incident r.f. powers. Although only a small change in the intensity ratio are caused by $\mathrm{He}$ addition when the plasma is operated at a relatively low r.f. power, the ratios are varied to a large extent under higher power conditions. Accordingly, the supplied power plays an important role in determining the plasma temperature in the $\mathrm{Ar}-\mathrm{He}$ mixed gas ICP.

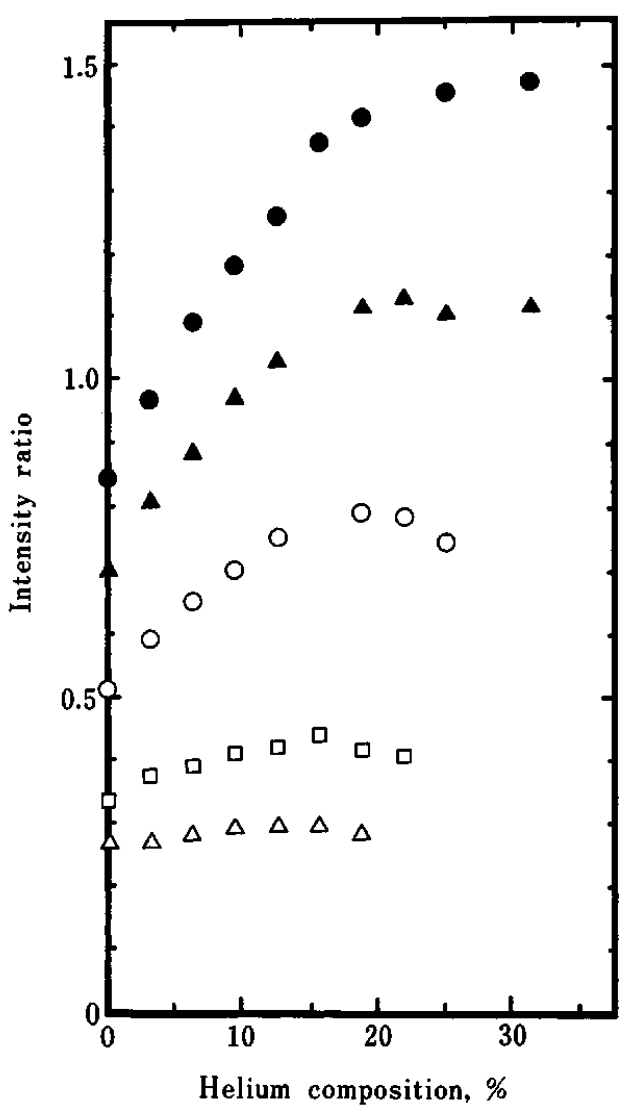

Fig. 4 Variations in the intensity ratio (Zn II 202.6/ Zn I 213.9) as a function of the helium composition for the r.f. power: $2.2 \mathrm{~kW}(\Theta), 1.9 \mathrm{~kW}(\Delta), 1.6 \mathrm{~kW}(\bigcirc), 1.3 \mathrm{~kW}(\square)$ and $1.1 \mathrm{~kW}(\Delta)$. 


\section{Correlation between argon FWHMs and zinc emission intensities}

As already described, the spectral width of the argon line hardly changed upon the addition of helium, while the intensities or the ionic-to-atomic ratio of the zinc lines increased. We consider that this type of behavior is principally due to a difference in the portions from which the argon and zinc lines are emitted.

The central channel of the plasma where the sample solutions are aspirated is a major position for the emission of the analyte species (zinc). In our experiment we selected the observation height to be $14 \mathrm{~mm}$ above the load coil (ALC), at which the normal analytical zone $^{12}$ of the ICP can be observed. On the other hand, the argon line provides information regarding the induction zone ${ }^{12}$ of the ICP. It can be deduced from the analysis on the argon line widths that the properties of the plasma core (induction zone) are almost independent of the He compositions in the ICP. Thus, the obtained results concerning the zinc intensity ratios can not be explained from the characteristics of the plasma core, itself.

The chemical species introduced into the plasma indirectly receive energies from the incident r.f. power through the induction zone surrounding the central channel. Therefore, the ability to transmit energies from the load coil has to be taken into consideration in the excitation of the species. It should be noted that the thermal conductivity of helium is about ten-times larger than that of argon. ${ }^{14}$ Though helium gas is incapable of the plasma modifications in the Ar-matrix ICP when the helium composition is less than $30 \%$, helium may serve as an energy carrier from the induction zone to the central portion of the ICP. These effects can lead to an increase in the plasma temperature at the central zone of the plasma, thus enhancing excitation of the zinc species.

\section{Behavior of background levels}

Figure 5 indicates the variations in the background level measured at $206.2 \mathrm{~nm}$ as a function of the helium content. It is found that the background levels decreases with an increase in the mixed amounts of helium. These results agree with those reported in a previous report. ${ }^{8}$

The continuum background of the ICP results primarily from radiative recombinations between argon ions and fast electrons. ${ }^{10}$ In this reaction, the kinetic energies of fast electrons are released as photons having a certain range of energies.

It is obvious that the background levels are lowered upon decreasing the incident r.f. power, mainly because the electron density in the ICP is decreased. However, as already described, the electron density scarcely change in the Ar-He mixed gas ICP. Much greater energies can be transferred through elastic collisions between helium atoms and fast electrons, compared to that in argon collisions, indicating that fast electrons are decelerated more effectively in the presence of helium. The reduction in the background levels may probably be

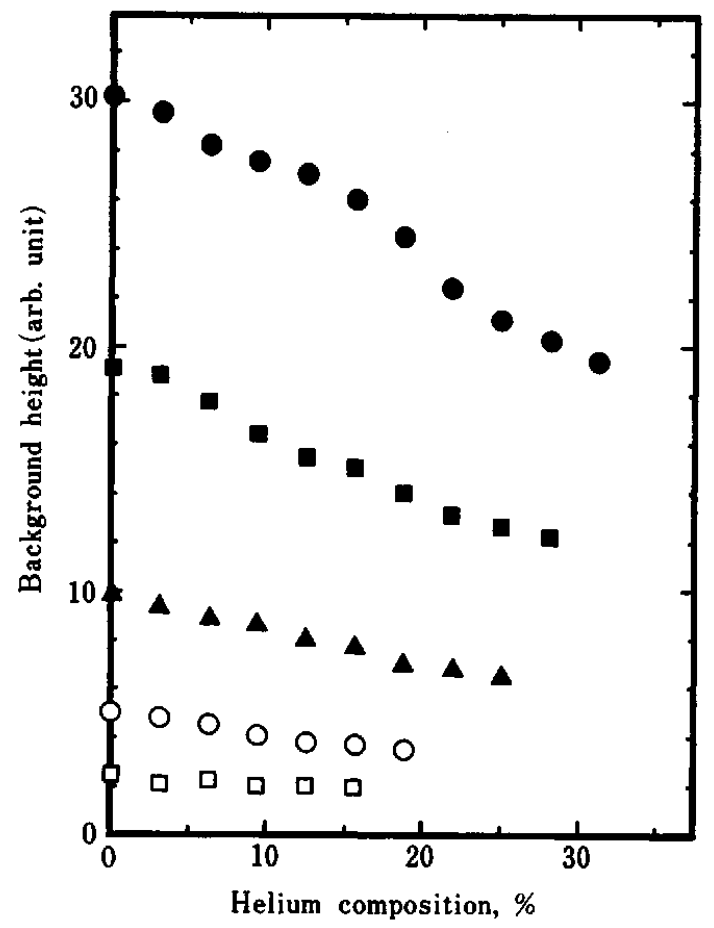

Fig. 5 Plots of the background height measured at $206.0 \mathrm{~nm}$ against the helium content in the outer gas flow for the r.f. power: $2.2 \mathrm{~kW}(\Theta), 1.9 \mathrm{~kW}(\square), 1.6 \mathrm{~kW}(\Delta), 1.3 \mathrm{~kW}(O)$ and $1.1 \mathrm{~kW}(\square)$.

related to variations in the energy distribution of free electrons in the plasma caused by helium addition. As described in Figs. 4 and 5, the effect of helium appears only at high incident r.f. powers, implying that the number of energetic electrons is dominantly reduced as a decrease in the supplied power.

\section{References}

1. S. Greenfield, J. L. Jones and G. T. Berry, Analyst [London], 89, 713 (1964).

2. S. Greenfield, H. McD. McGreachin and P. B. Smith, Talanta, 22, 1 (1975).

3. D. Truitt and J. W. Robinson, Anal. Chim. Acta, 50, 61 (1970).

4. A. Montaser and J. Mortazavi, Anal. Chem., 52, 225 (1980).

5. A. Montaser, V. A. Fassel and J. Zalewski, Appl. Spectrosc., 35, 292 (1981).

6. E. H. Choot and G. Horlick, Spectrochim. Acta, 41B, 889 (1986).

7. E. H. Choot and G. Horlick, Spectrochim. Acta, 41B, 925 (1986).

8. E. H. Choot and G. Horlick, Spectrochim. Acta, 41B, 907 (1986).

9. B. S. Sheppard, J. A. Caruso, K. A. Wolnik and F. L. Fricke, Appl. Spectrosc., 44, 712 (1990).

10. J. M. Mermet, "Inductively Coupled Plasma Emission Spectroscopy", ed. P. W. J. M. Boumans, Part II, Chap. 10, John Wiley \& Sons, New York, 1987. 
11. H. R. Griem, “Plasma Spectroscopy”, McGraw-Hill, New York, 1964.

12. S. R. Koirtyohann, J. S. Jones, C. P. Jester and D. A. Yates, Spectrochim. Acta, 36B, 53 (1981).

13. B. L. Caughlin and M. W. Blades, Spectrochim. Acta, 40B, 98 (1985).
14. Y. S. Touloukian, "Thermophysical Properties of Matter", Vol. 1, Plenum Press, New York, 1970.

(Received August 7, 1992) (Accepted October 28, 1992) 